\title{
An insight of rapamycin against cadmium's neurotoxicity
}

\author{
Chong $\mathrm{Xu}$, Shile Huang and Long Chen
}

Cadmium $(\mathrm{Cd})$, a toxic environmental pollutant, has a long biological half-life (15-20 years) and thus can be readily accumulated in human body with age. Due to its high permeability to blood-brain barrier, $\mathrm{Cd}$ can evoke overproduction of reactive oxygen species (ROS) and consequently induce neuronal cell death in brain. $\mathrm{Cd}$ has been regarded as a possible etiological factor for human neurodegenerative diseases, such as Parkinson's disease, Alzheimer's disease, and Huntington's disease [1]. Thus, it is of great importance to find effective interventions against $\mathrm{Cd}$-induced oxidative stress in the central nervous system.

Rapamycin is a potent and specific inhibitor of mammalian/mechanistic target of rapamycin (mTOR) [2]. Abundant evidence suggests that rapamycin may possess neuroprotective effect on various neurological diseases [2]. Our recent studies have revealed that pretreatment with rapamycin prevents $\mathrm{Cd}$-induced neuronal cell death, in part by attenuating Cd-induced ROS activation of mTOR signaling pathways in vitro and in vivo [3-5]. In addition, we have noticed that $\mathrm{Cd}$-induced ROS can activate c-Jun N-terminal kinase (JNK) and extracellular signal-regulated kinase $1 / 2$ (Erk1/2), partly contributing to neuronal apoptosis [6]. It has been shown that rapamycin impacts the activity of JNK and Erk1/2 under different conditions [7]. This prompted us to study whether and how rapamycin mitigates $\mathrm{Cd}$ neurotoxicity by preventing ROS from activation of JNK and Erk1/2 pathways.

The mitochondrion is not only the factory of energy but also the main generator of ROS in a cell. First of all, we investigated whether rapamycin prevents $\mathrm{Cd}$ from inducing ROS in the mitochondria of neuronal cells. For this, we employed thenoyltrifluoroacetone (TTFA), a mitochondrial complex II ubiquinone site inhibitor that blocks electron supply to ubiquinol and consequentially limits the formation of ubisemiquinone, and antimycin $\mathrm{A}$, a mitochondrial complex III inhibitor that increases the lifetime of ubisemiquinone. Our results showed that cotreatment with rapamycin/TTFA inhibited Cd-evoked ROS more potently than treatment with rapamycin or TTFA alone in PC12 cells and mouse primary neurons, whereas treatment with antimycin A alone markedly triggered ROS, and further enhanced Cd-induced ROS, which could be repressed by rapamycin pretreatment in the cells [8]. Furthermore, Mito-TEMPO, a mitochondria-targeted antioxidant, significantly strengthened the inhibitory effects of rapamycin on Cd-induced ROS and neuronal apoptosis [8]. Hence, our results indicate that rapamycin attenuates $\mathrm{Cd}$-induced neuronal apoptosis indeed by preventing Cd-elicited mitochondrial ROS.

Next, we tested whether rapamycin attenuates $\mathrm{Cd}$ neurotoxicity by blocking Cd-induced mitochondrial ROS activation of JNK and Erk1/2 pathways. Our data showed that rapamycin remarkably suppressed Cdinduced phosphorylation of JNK and Erk1/2 in PC12 cells and primary neurons, which was strengthened by addition of Mito-TEMPO [8]. To validate the involvement of JNK and Erk1/2 in rapamycin's protection against Cdinduced neuronal apoptosis, SP600125 (JNK inhibitor) and U0126 (MEK1/2 inhibitor) were utilized. We found that SP600125 and U0126, respectively, potentiated rapamycin's prevention from $\mathrm{Cd}$-induced cell apoptosis [8]. Consistently, using recombinant adenoviruses expressing dominant negative c-Jun (dn-c-Jun) or MKK1 (MKK1-K97M), we observed that ectopic expression of dn-c-Jun or MKK1-K97M also potently improved the inhibitory effect of rapamycin on Cd neurotoxicity [8]. Furthermore, pretreatment with SP600125 or U0126, or expression of dn-c-Jun or MKK1-K97M enhanced the inhibitory effect of rapamycin or Mito-TEMPO on Cdinduced ROS [8]. Collectively, these results strongly suggest that rapamycin blocks Cd-induced mitochondrial

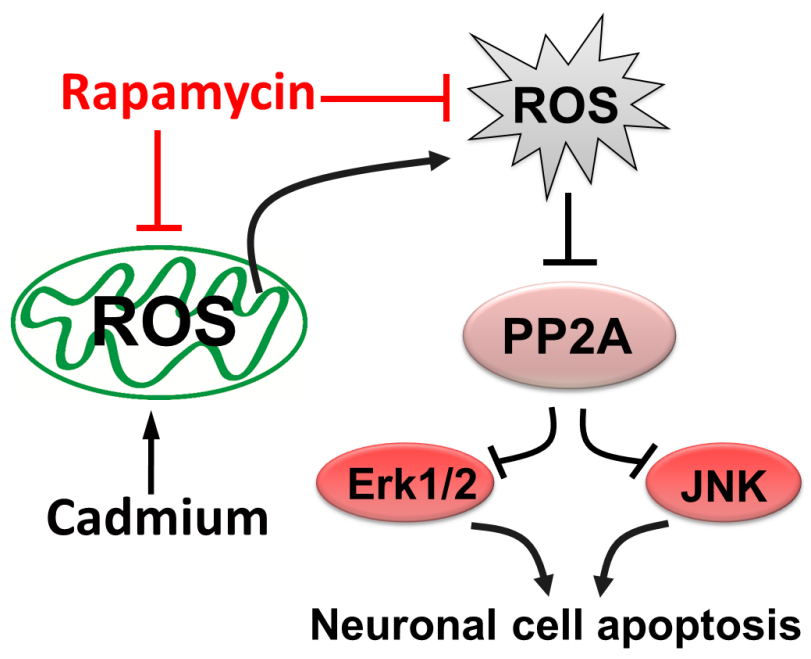

Figure 1: Schematic diagram of rapamycin against cadmium's neurotoxicity. Rapamycin prevents Cd-evoked mitochondrial ROS inactivation of PP2A, thus suppressing activation of JNK and Erk1/2 pathways, and rescuing neuronal cell apoptosis. 
ROS activation of JNK and Erk1/2 pathways, thereby rescuing against neuronal apoptosis triggered by $\mathrm{Cd}$.

Finally, we studied how rapamycin prevents $\mathrm{Cd}$ induced mitochondrial ROS from activating JNK and Erk1/2 pathways. It is known that PP2A negatively regulates JNK and Erk1/2, in response to stress response [6], and PP2A can be activated by rapamycin [7]. So, we examined whether rapamycin blocks $\mathrm{Cd}$ activation of JNK and Erk1/2 pathways by preventing $\mathrm{Cd}$-induced mitochondrial ROS from inactivation of PP2A. The results showed that $\mathrm{Cd}$-induced elevation of demethylation and phosphorylation of PP2A catalytic subunit, two events related to PP2A inactivation, was substantially suppressed by rapamycin or Mito-TEMPO alone, and more potently inhibited by their co-treatment in PC12 cells and primary neurons [8]. To corroborate the above findings, genetic recue experiments for $\mathrm{PP} 2 \mathrm{~A}$ were carried out. We observed that over-expression of wild-type $\mathrm{PP} 2 \mathrm{~A}$ reinforced rapamycin or Mito-TEMPO suppression of activated JNK and Erk1/2 pathways, as well as ROS production and apoptosis in PC12 cells in response to Cd [8]. Therefore, these data support that rapamycin ameliorates $\mathrm{Cd}$-induced neuronal apoptosis by preventing mitochondrial ROS inactivation of PP2A, thus blocking activation of JNK and Erk1/2 cascades.

In conclusion, we have demonstrated that rapamycin prevents Cd-evoked mitochondrial ROS inactivation of PP2A, thus suppressing activation of JNK and Erk1/2 pathways, and rescuing neuronal apoptosis (Figure 1). The new finding highlights rapamycin as a promising agent for prevention of $\mathrm{Cd}$-induced oxidative stress and neurodegenerative diseases.
Long Chen: Jiangsu Key Laboratory for Microbes and Functional Genomics, Jiangsu Key Laboratory for Molecular and Medical Biotechnology, College of Life Sciences, Nanjing Normal University, Nanjing, P. R. China

Correspondence to: Long Chen, email Ichen@njnu.edu.cn

Keywords: rapamycin; cadmium; ROS; neuronal cells; PP2A; JNK; Erk1/2; Neuroscience

Received: December 02, 2016

Published: January 28, 2017

\section{REFERENCES}

1. Monroe RK, and Halvorsen SW. Free Radic. Biol. Med. 2006; 41:493-502.

2. Bove J, et al. Nat. Rev. Neurosci. 2011; 12:437-452.

3. Chen L, et al. J. Neurochem. 2008; 105:251-261.

4. Chen L, et al. Free Radic. Biol. Med. 2011; 50:624-632.

5. Chen S, et al. Neuropathol. Appl. Neurobiol. 2014; 40:759777.

6. Chen L, et al. Free Radic. Biol. Med. 2008; 45:1035-1044.

7. Huang S, et al. Mol. Cell. 2003; 11:1491-1501.

8. Xu C, et al. Neuropharmacology. 2016; 105:270-284. 